\title{
DETERMINAÇÃO DA AUTENTICIDADE DE AMOSTRAS DE AZEITE COMERCIAIS APREENDIDAS NO ESTADO DO ESPÍRITO SANTO USANDO UM ESPECTROFOTÔMETRO PORTÁTIL NA REGIÃO DO NIR
}

Priscilla C. Santos ${ }^{\mathrm{a}}$, Flavia Tosato ${ }^{\mathrm{b}}$, Mirelly Cesconetto ${ }^{\mathrm{a}}$, Thayná Corrêa ${ }^{\mathrm{a}}$, Francine D. Santos ${ }^{\mathrm{b}}$, Valdemar Lacerda Jr. ${ }^{\mathrm{b}}$, André A. Pires ${ }^{\mathrm{a}}$, Araceli V. F. N. Ribeiro ${ }^{\mathrm{a}}$, Paulo R. Filgueiras ${ }^{\mathrm{b}, \#}$ e Wanderson Romã $0^{\mathrm{a}, \mathrm{b}, \mathrm{c}, *, \mathbb{1}}$

anstituto Federal do Espírito Santo, 29106-010 Vila Velha - ES, Brasil

bDepartamento de Química, Universidade Federal do Espírito Santo, 29075-910 Vitória - ES, Brasil

'Instituto Nacional de Ciência e Tecnologia Forense (INCT), Vitória - ES, Brasil

Recebido em 02/02/2020; aceito em 06/04/2020; publicado na web em 20/05/2020

\begin{abstract}
DETERMINATION OF THE AUTHENTICITY OF COMMERCIAL OLIVE OIL SAMPLES SEIZED IN THE ESPÍRITO SANTO STATE USING A PORTABLE SPECTROPHOTOMETER IN THE NIR REGION. Many instrumental techniques are used to monitor the quality of food products, highlighting near infrared spectroscopy (NIRS). In order to determine the authenticity of 60 commercial oil samples seized by the Espírito Santo state police, this work used a portable spectrometer in the NIR region associated with chemometric tools such as principal component analysis (PCA) and partial least squares regression (PLS) to classify and quantify possible adulterants present such as soybean oil. In addition, physicochemical tests (acidity, peroxide and ultraviolet absorption) were carried out on commercial samples. The PLS regression model built to quantify soybean oil showed a coefficient of determination $\left(\mathrm{R}^{2}\right)$ greater than 0.99 , showing itself to be satisfactory. The results showed that all samples analyzed had a high content of adulterant and cannot be classified as extra virgin olive oil.
\end{abstract}

Keywords: NIR; chemometrics; olive oil; soy oil.

\section{INTRODUÇÃO}

A apreensão de produtos alimentícios com suspeita de falsificação aumentou significativamente nos últimos anos, em decorrência do crescente número de casos de adulteração de alimentos, acarretando assim, em uma maior busca de controle e fiscalização. ${ }^{1,2}$

A preocupação dos consumidores e órgãos fiscalizadores quanto à adulteração e fraude alimentar é que estas, embora praticadas desde os tempos remotos, se tornaram mais aprimoradas e minuciosas nos últimos anos. ${ }^{3}$ Devido a pouca possibilidade de descoberta, a fraude de alimentos tornou-se um problema emergente que causa impactos negativos, não só para a saúde do consumidor, mas também por acarretar prejuízos econômicos, sociais e ambientais., ${ }^{4,5}$

O Brasil é um dos maiores produtores de alimentos do mundo e também encara a problemática da falsificação destes. Um exemplo disso é a adulteração do azeite de oliva extravirgem (AOEV), o qual é um produto com alto valor nutricional e econômico que pode ser modificado através da adição de óleos vegetais de menor valor comercial, como o óleo de soja. ${ }^{6}$

Os órgãos nacionais que apresentam normas e diretrizes para o controle de qualidade do AOEV são a Agência Nacional de Vigilância Sanitária (ANVISA) e o Ministério da Agricultura, Pecuária e Abastecimento (MAPA). ${ }^{7,8}$ Apenas no ano de 2019, o MAPA proibiu a venda de mais de 30 marcas de AOEV, sendo esse número aumentado quando somados às apreensões de anos anteriores. ${ }^{9}$ Isto implica em vários malefícios, haja vista que o Brasil é um grande importador desse produto. ${ }^{10}$

A ANVISA e o MAPA impõem a determinação de quatro parâmetros físico químicos para o monitoramento das propriedades físico-químicas do AOEV: acidez, índice de peróxido, absorbância no ultravioleta e análise sensorial. Porém, com o intuito de melhorar a fiscalização, vários métodos analíticos com maior sensibilidade e

*e-mail: wandersonromao@gmail.com

\#e-mail alternativo: filgueiras.pr@gmail.com seletividade vêm sendo utilizados na investigação de adulteração alimentar. Analisando na literatura, o número de artigos científicos publicados na última década (2010-2020, Figura 1), é possível mostrar que as principais técnicas utilizadas na análise e controle de qualidade de alimentos são: a cromatografia líquida de alta eficiência (CLAE), ${ }^{11-22}$ cromatografia gasosa (CG),${ }^{23-28}$ espectroscopia na região do ultravioleta (UV/VIS), ${ }^{23,29-35}$ espectroscopia na região do infra vermelho próximo, near infrared spectroscopy (NIRS), ${ }^{36-54}$ espectroscopia na região do infravermelho médio, mid infrared spectroscopy (MIRS), ${ }^{55-62}$ espectroscopia Raman, ${ }^{40,52,63-74}$ ressonância magnética nuclear $(\mathrm{RMN})^{33,75-86}$ e narizes eletrônicos (electronic noses, E-noses)..$^{50,87-91}$

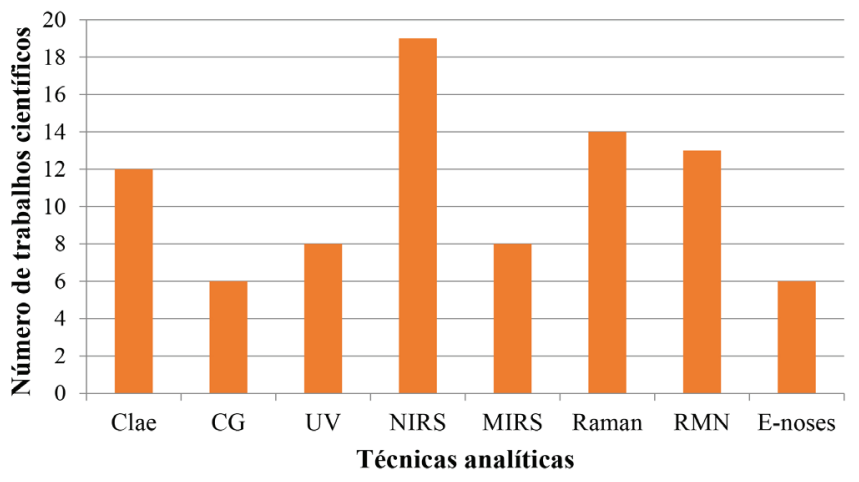

Figura 1. Número de trabalhos científicos envolvendo técnicas analíticas na análise de alimentos entre os anos de 2010 - 2020

Lohumu et al., em 2015, enfatizaram que dentre as técnicas espectroscópicas vibracionais (infravermelho próximo, infravermelho médio, espectroscopia Raman e imagens hiperespectrais) a NIRS, combinada com ferramentas quimiométricas, destaca-se no que diz respeito a análise de alimentos. ${ }^{92} \mathrm{~A}$ Tabela 1 apresenta importantes aplicações, ao longo de vários anos, enfatizando o emprego da NIRS com ênfase no controle de qualidade de óleos comestíveis. ${ }^{93-104}$ 
A NIRS fornece informações espectrais baseadas em sobretons e bandas de combinação de grupos funcionais específicos. ${ }^{105}$ Esses espectros são gerados a partir das vibrações de moléculas que possuem fortes interações entre seus átomos, as quais possuem, em geral, átomos de $\mathrm{C}, \mathrm{N}$ e $\mathrm{O}$ ligados ao átomo de $\mathrm{H} .{ }^{106}$ Por esse motivo, a NIRS é amplamente empregada na análise de moléculas orgânicas sendo capaz de fornecer ricas informações acerca da composição de um produto alimentar. ${ }^{107-108}$

No que tange à determinação de adulteração em amostras de AOEV, a NIRS possui grande aplicação. ${ }^{109}$ As principais regiões que caracterizam o AOEV podem se encontrar nas faixas de $1140-1250 \mathrm{~nm}$ e 1350-1500 nm devido à absorção de grupos funcionais de ácidos graxos livres e moléculas insaturadas, os quais são abundantes em óleos. ${ }^{110}$ Também foi relatado que compostos presentes no AOEV como clorofila, carotenóides e ácido oleico absorvem na região de 450 $\mathrm{nm}, 670 \mathrm{~nm}$ e $1725 \mathrm{~nm},{ }^{11-113}$ respectivamente. É possível encontrar diversos artigos na literatura relacionados ao uso da NIRS à qualidade do azeite evidenciando a eficácia dessa técnica.

Em 2016, Karunathilaka et al. utilizaram a NIR como uma ferramenta de triagem rápida e direcionada para autenticidade de produtos de AOEV obtendo como resultado uma diferença significativa entre os espectros do AOEV daqueles adulterados nos comprimentos de 5150 a $5350 \mathrm{~cm}^{-1}$, viabilizando a utilização da NIRS para este tipo de análise. ${ }^{114}$ Em 2018, Vanstone et al. desenvolveram um método utilizando a NIRS associada a quimiometria, PCA (análise de componentes principais), para detectar adulteração em AOEV com outros tipos de óleos comestíveis, são estes: óleo de soja, milho, girassol e canola; que não necessita de preparação de amostra e podendo ser concluído em menos de 10 minutos. O estudo em questão determinou as porcentagens mais baixas de detecção para os adulterantes citados anteriormente, obtendo como resultado porcentagens, em massa, de aproximadamente $20 \%$ para óleos de milho e girassol, $15 \%$ para óleo de soja e de $10 \%$ para óleo de canola, apresentando bons limites de detecção para o método NIR. ${ }^{115} \mathrm{Em} 2018$, Willenberg et al. desenvolveram modelos quimiométricos para predizer, através da NIRS, os parâmetros físico-químicos como acidez, índice de peróxido, $\mathrm{K}_{232} \mathrm{e}$ $\mathrm{K}_{270}$, índice de p-Anisidin, pirofeofitina A e diacilgliceróis isoméricos, associando esses parâmetros à análise sensorial. Adicionalmente, nesse estudo também foi possível prever o grau de envelhecimento do AOEV. ${ }^{116}$ Além desses, em 2019, Yan, Stuijvenberg e Ruth, também utilizaram a NIRS, a fim de classificar amostras AOEV em função de seus dados composicionais, utilizando agora um equipamento portátil. Como resultado, o estudo revelou como promissor a utilização do NIR portátil, uma vez que foi capaz de diferenciar 130 amostras comerciais de azeites. ${ }^{17}$

Os equipamentos miniaturizados e portáteis estão sendo desenvolvidos para suprirem o custo elevado de manutenção, alta complexidade e difícil operação que muitas técnicas analíticas possuem. Exemplo disso é o micro espectrômetro portátil na região do NIR (MicroNIR). Esse possibilita uma análise in loco sem destruição da amostra com custo e tempo reduzidos. ${ }^{118,119} \mathrm{O}$ emprego da NIRS portátil na análise de alimentos já tem mostrado muitos resultados satisfatórios, inclusive na análise de óleos. Em 2017, Basri et al. utilizaram o MicroNIR na análise de óleo de palma com o objetivo de diferenciar amostras puras de amostras adulteradas com banha de porco, sendo que o modelo construído possibilitou a detecção do teor deste adulterante abaixo de $0,5 \mathrm{wt} \% .{ }^{120}$ A qualidade do óleo de palma também foi avaliada por Kaufmann et al. em 2019, em que a acidez foi determinada nas amostras utilizando a NIRS portátil. ${ }^{121}$ Oliveira et al., em 2020, conseguiram determinar a autenticidade do colorau em pó adulterado com amido de batata, goma arábica e urucum utilizando a NIRS portátil. ${ }^{122}$ Também utilizando essa técnica, Pan et al., em 2015, avaliaram o teor de umidade, sólidos solúveis, sacarose assim como propriedades mecânicas em amostras de beterraba. ${ }^{123} \mathrm{~A}$ NIRS portátil também foi utilizada em estudos realizados em café, leite e carne. ${ }^{124-126}$

No que tange a análise de café, em 2018, Correia et al utilizou a técnica NIR portátil a fim de avaliar a qualidade do café Arábica através da quantificação de adulterações. Para isso, foram produzidas 125 amostras contendo um total de treze concentrações distintas de adulterante, variando de 1 a 100 wt\%. ${ }^{124}$ Já para análise de amostas de leite, também em 2018, Liu et al. estudaram a evolução da técnica NIR portátil para autenticação do leite orgânico comparando com o NIR de bancada, avaliando 37 amostras de leites orgânicos de varejo. Eles obtiveram um resultado semelhante entre as duas técnicas. ${ }^{125}$ Já em 2020, Savoia et al. estudaram a qualidade da carne diretamente do abatedouro utilizando o NIR portátil, analisando músculo bovino e carcaças de touros jovens, obtendo resultados satisfatórios. ${ }^{126}$

Assim como nos estudos apresentados, a NIRS portátil possui grande utilidade no que diz respeito à avaliação da autenticidade do AOEV, pois este se trata de um produto que é alvo frequente de adulterações e necessita de análises rápidas, de baixo custo e confiáveis. Entretanto, apesar da ampla aplicação, existem poucos relatos na literatura do uso da NIRS portátil para o emprego de amostras comerciais que são suspeitas de fraude. Por esse motivo, o presente trabalho, em parceria com a Polícia Civil do Estado do Espírito Santo, utilizou o microNIR associado aos modelos quimiométricos como PCA e PLS (regressão por mínimos quadrados parciais) a fim de determinar a autenticidade de 60 amostras comerciais de AOEV e predizer a quantidade de óleo de soja presente nas mesmas. Nessas amostras, foram também avaliados os parâmetros físico-químicos como: acidez, índice de peróxido e absorbância no ultravioleta.

\section{METODOLOGIA}

\section{Amostras e reagentes}

Um total de 60 amostras de azeite comercial, das marcas Anna ${ }^{\circledR}$, Fazenda Herdade ${ }^{\circledR}$, Oliveiras do Conde ${ }^{\circledR}$, Porto Galo ${ }^{\circledR}$ e Serra de Montejunto ${ }^{\circledR}$, provenientes de diferentes estabelecimentos de coleta, foram fornecidos pela Polícia Civil do Estado do Espírito Santo (PC-ES). Para uma das marcas foram apreendidas amostras do tipo AOEV e óleo composto contendo uma mistura 51:49 wt\% AOEV:óleo de soja. Para as demais marcas foram apreendidas apenas amostras de AOEV. As marcas foram aletoriamente nomeadas de A - E conforme apresentado na Tabela 2 juntamente com as informações descritas nos rótulos das mesmas. Além disso, para a elaboração do modelo de classificação quimiométrico, outras amostras foram também estudadas como: amostras comerciais de AOEV (marcas Olive ${ }^{\circledR}$, Olisur ${ }^{\circledR}$, Santiago $^{\circledR}$, Chile $^{\circledR}$; O-live ${ }^{\circledR}$ (referência), Midas ${ }^{\circledR}$, Komili $^{\circledR}$, Cocinero $^{\circledR}$, Borges $^{\circledR}$, Nova oliva $^{\circledR}$, Ecolive $^{\circledR}$ e Gallo ${ }^{\circledR}$ ); óleo vegetal de soja (da marca Liza ${ }^{\circledR}$, Cargill agrícolas S A, Mairinque, Brasil) e óleos compostos (Dom João ${ }^{\circledR}-30$ wt\% de AOEV; Maria ${ }^{\circledR}$ - 12 wt $\%$ de AOEV, Vittoria - 51 wt $\%$ de AOEV; e Faisão ${ }^{\circledR}$ - 5 wt $\%$ de AOEV).

Todos os reagentes utilizados (clorofórmio, éter etílico, ambos da marca Hexis ${ }^{\circledR}$, Indaiatuba, Brasil); álcool etílico, cicloexano, ácido acético, iodeto de potássio, fenolftaleína, e tiossulfato de sódio $\left(\right.$ Neon $^{\circledR}$, Suzano, Brasil) e hidróxido de sódio (Dinâmica ${ }^{\circledR}$, Indaiatuba, Brasil) possuíam grau analítico.

Para o desenvolvimento do método de quantificação de óleo de soja em amostras de AOEV, misturas binárias foram produzidas, cada concentração em triplicata, a partir da pesagem de uma massa total de $4 \mathrm{~g}$ de AOEV com 19 concentrações de óleo vegetal $(0,1,3$, $5,10,15,20,25,30,35,40,45 ; 50 ; 55 ; 60 ; 70 ; 80 ; 90$ e $100 \mathrm{wt} \%)$. 
Tabela 1. Aplicações da NIRS na análise e controle de qualidade de óleos relatadas na literatura.

\begin{tabular}{|c|c|c|c|c|c|}
\hline Ano & Aplicação & Instrumento utilizado & $\begin{array}{c}\text { Faixa/ região } \\
\text { espectral }\end{array}$ & $\begin{array}{l}\text { Modelo quimiométrico } \\
\text { empregado }\end{array}$ & Referência \\
\hline 1994 & Classificação de óleos vegetais & InfraAlyzer 500 & $1600-2200 \mathrm{~nm}$ & PCA & Sato $^{93}$ \\
\hline 1996 & Detecção de adulteração em AOEV & NIRSystems 6500 & $1100-2498 \mathrm{~nm}$ & PCA & Wesley et al..$^{94}$ \\
\hline 1998 & Avaliação da qualidade de óleo e gordura de fritura & $\begin{array}{l}\text { FT-NIR universal } \\
\text { spectrometer (NIRVIS) }\end{array}$ & $10000-4000 \mathrm{~cm}^{-1}$ & PLS & Hein et al..$^{95}$ \\
\hline 2002 & Detecção de adulteração em AOEV & NIRSystems 6500 & $1100-2498 \mathrm{~nm}$ & PLS & Downey et al. ${ }^{96}$ \\
\hline 2005 & Avaliação da qualidade de óleo de peixe & NIRSystems 5000 & $1100-2500 \mathrm{~nm}$ & PCA, PLS & Cozzolino et al..$^{97}$ \\
\hline 2005 & Avaliação da qualidade de óleos comestíveis & Nicolet 870 spectrometer & $8000-4000 \mathrm{~cm}^{-1}$ & PCA, PLS & Yang et al. ${ }^{98}$ \\
\hline 2008 & Avaliação da qualidade de óleos comestíveis & Spectrum GX FT-NIR & $6134-3068 \mathrm{~cm}^{-1}$ & PLS & Pereira et al. \\
\hline 2013 & Avaliação da qualidade de AOEV & MPA FT-NIR & $12500-4000 \mathrm{~cm}^{-1}$ & PLS & $\begin{array}{l}\text { Inerejos-García } \\
\quad \text { et al. } .^{99}\end{array}$ \\
\hline 2017 & $\begin{array}{c}\text { Detecção da quantidade de } \alpha \text {-tocoferol e tocoferóis } \\
\text { totais em AOEV }\end{array}$ & Luminar 5030 & $1100-2300 \mathrm{~nm}$ & PCA, PLS & Cayuela et al. ${ }^{100}$ \\
\hline 2018 & Detecção de adulteração em óleo de gergelim & Antaris II FT-NIR & $10000-4500 \mathrm{~cm}^{-1}$ & PLS & Chen et al..$^{101}$ \\
\hline 2018 & Detecção de adulteração em óleo de girassol & $\begin{array}{l}\text { FT-NIR Spectrometer - } \\
\text { MATRIX-F } 144\end{array}$ & $833-2300 \mathrm{~nm}$ & PLS & Picouet et al. ${ }^{102}$ \\
\hline 2019 & Detecção de adulteração em óleo de argan & AVS-USB 2000 & $1000-1700 \mathrm{~nm}$ & PCA, PLS & Farres et al..$^{103}$ \\
\hline 2020 & Avaliação da qualidade de AOEV & $\begin{array}{l}\text { Buchi } 137 \text { NIRFlex } \\
\text { N-500 }\end{array}$ & $10000-4000 \mathrm{~cm}^{-1}$ & PLS & Mustorgi et al. ${ }^{104}$ \\
\hline
\end{tabular}

Tabela 2. Valores de acidez, peróxido e extinção específica descritos nos rótulos das marcas analisadas, assim como quantidade de amostras para cada marca

\begin{tabular}{|c|c|c|c|c|c|c|}
\hline Marca & Acidez & Peróxido & Extinção específica a 270 & Extinção específica a 232 & delta $\mathrm{K}$ & Quandidade de amostras \\
\hline$A-(E V)$ & $<0,4 \%$ & $\leq 20 \mathrm{meq} / \mathrm{kg}$ & $\leq 0,22$ & $\leq 2,5$ & $\leq 0,01$ & 14 \\
\hline $\mathrm{B}-(\mathrm{EV})$ & $<0,5 \%$ & $\leq 20 \mathrm{meq} / \mathrm{kg}$ & $\leq 0,22$ & $\leq 2,5$ & $\leq 0,01$ & 20 \\
\hline$B-(51 \%)$ & - & - & - & - & - & 3 \\
\hline $\mathrm{C}-(\mathrm{EV})$ & $<0,2 \%$ & $\leq 20 \mathrm{meq} / \mathrm{kg}$ & $\leq 0,22$ & $\leq 2,5$ & $\leq 0,01$ & 5 \\
\hline $\mathrm{D}-(\mathrm{EV})$ & $<0,4 \%$ & $\leq 20 \mathrm{meq} / \mathrm{kg}$ & $\leq 0,22$ & $\leq 2,5$ & $\leq 0,01$ & 6 \\
\hline$E-(E V)$ & $<0,2 \%$ & $\leq 20 \mathrm{meq} / \mathrm{kg}$ & $\leq 0,22$ & $\leq 2,5$ & $\leq 0,01$ & 12 \\
\hline
\end{tabular}

\section{Análises físico-químicas}

Acidez

Compreende-se o índice de acidez como um ensaio volumétrico para avaliar a qualidade e a pureza do azeite. Com esse teste, é possível avaliar se o azeite esteve em contato com água durante o processo de fabricação, uma vez que determina o teor de ácidos graxos livres resultantes de reações de hidrólise, por meio da porcentagem de ácido oleico. Dessa forma, quanto maior o índice de acidez, maior o nível de deterioração do azeite. ${ }^{127,128} \mathrm{~A}$ legislação determina um valor de acidez máximo de $0,8 \mathrm{wt} \%$ de ácido oleico para AOEV.

Para a análise do índice de acidez das amostras de azeite comercial, a norma do Instituto Adolfo Lutz foi utilizada como referência. ${ }^{129}$ Assim, uma massa de 2 gramas de amostra foi pesada em um erlenmeyer de $125 \mathrm{~mL}$, adicionando-se $25 \mathrm{~mL}$ de solução éter-álcool etílico (2:1) neutra e 3 gotas de fenolftaleína. Após, a titulação foi efetuada com solução de $\mathrm{NaOH} 0,01 \mathrm{~mol} \mathrm{~L}^{-1}$.

Todas as análises foram realizadas em triplicata, e os valores de acidez (\% de ácido oleico) foram calculados de acordo com a Equação 1, onde $\mathrm{V}$ corresponde ao volume de hidróxido de sódio gasto na titulação (L), C é a concentração da solução de hidróxido de sódio (dado em $\mathrm{mol} \mathrm{L}^{-1}$ ), M é a massa molar do ácido oleico (em $\mathrm{g} \mathrm{mol}^{-1}$ ) e $\mathrm{m}$ corresponde a massa da amostra (em g).

$$
\text { acidez }=\frac{V \times C \times M}{10 \times m}
$$

\section{Peróxido}

Determinado de acordo com o anexo III do regulamento da Comissão Europeia n $n^{\circ} 2568$ de 11 de julho de 1991, ${ }^{130}$ o índice de peróxido trata-se de um teste volumétrico utilizado para determinar a oxidação inicial, a rancidez e deterioração que pode ter ocorrido nos antioxidantes naturais presentes no azeite. ${ }^{131} \mathrm{~A}$ legislação estabelece um limite de $20 \mathrm{meq} / \mathrm{kg}$ para AOEV.

Dessa forma, uma massa de $2,5 \mathrm{~g}$ de cada amostra foi pesada em um erlenmeyer de $250 \mathrm{~mL}$, sendo essa dissolvida em $8 \mathrm{~mL}$ de clorofórmio, $12 \mathrm{~mL}$ de ácido acético glacial e $0,5 \mathrm{~mL}$ de solução saturada de iodeto de potássio, e deixada por 1 minuto em repouso em ambiente isento de luz. Após, foram adicionados $15 \mathrm{~mL}$ de água destilada e 0,5 mL de solução saturada de amido, utilizado como indicador para posterior titulação.

Em sequência, titulou-se com solução de tiossulfato 0,01 N, além de um ensaio em branco, que consiste na titulação sem o analito. Com os dados obtidos nas titulações em triplicata, o índice de peróxido foi calculado conforme a Equação 2.

$$
\text { índice de peróxido }=\frac{(A-B) \times N \times 1000}{m}
$$

em que, A corresponde ao volume da solução de tiossulfato de sódio (L) gasto na titulação da amostra, B ao volume da solução de tiossulfato de sódio gasto na titulação do branco (L), $\mathrm{N}\left(\mathrm{m} \mathrm{E} \mathrm{g}^{-1} \mathrm{~L}^{-1}\right)$ à normalidade da solução de tiossulfato de sódio e $\mathrm{m}$ à massa da amostra $(\mathrm{g})$. 


\section{Absorbância no Ultravioleta}

A absorbância no ultravioleta é um exame espectrofotométrico para avaliar a qualidade do AOEV por informar sobre a identidade e estado de conservação do mesmo, além de indicar possíveis alterações advindas do processamento. Foi determinado segundo o anexo IX do regulamento da Comissão Europeia n ${ }^{\circ} 2568$ de 11 de julho de 1991. ${ }^{132}$

Assim, uma massa de aproximadamente $0,25 \mathrm{~g}$ de amostra foi dissolvida em $25 \mathrm{~mL}$ de cicloexano e foram lidas as absorbâncias em espectrofotômetro UV/VIS de feixe duplo modelo UV-vis Cary 60 da Agilent. Os coeficientes de extinção foram lidos nos comprimentos de onda de $232 \mathrm{~nm}, 270 \mathrm{~nm}$ e $\Delta \mathrm{K}$ foram calculados conforme a Equação 3.

$$
\mathrm{K}_{270}=\frac{A_{270}}{c l} \quad \mathrm{~K}_{232}=\frac{A_{232}}{c l} \quad \Delta \mathrm{K}_{232}=A_{270}-\frac{A_{266}+A_{274}}{2}
$$

$\mathrm{K}_{232}=$ Extinção especifica a $\lambda=232 \mathrm{~nm} ; \mathrm{K}_{270}=$ Extinção especifica a $\lambda=270 \mathrm{~nm} ; \Delta \mathrm{K}=$ Extinção específica no comprimento de onda para a máxima absorção em torno de $270 \mathrm{~nm} ; \mathrm{A}_{270}, \mathrm{~A}_{232}, \mathrm{~A}_{266}$ e $\mathrm{A}_{274}$ são absorvâncias, $c$ é a concentração do azeite em g/100 mL, e $l$ é o percurso óptico $(=1 \mathrm{~cm})$.

As absorbâncias foram adquiridas nos comprimentos de 232 e 270 $\mathrm{nm}$, por decorrência da presença de sistemas dienos e trienos conjugados, e os valores são datados em termos de extinção específica. ${ }^{131}$

\section{Espectroscopia na região do infravermelho próximo (NIR)}

A análise de espectroscopia de infravermelho foi realizada utilizando um instrumento modelo miniatura MicroNIR TM Pro 1700. Os dados foram obtidos do software 3.0 da Viavi Solutions Inc. Os espectros foram adquiridos na faixa de $908-1676 \mathrm{~nm}$, com varredura de 100 espectros, um tempo de integração de $8 \mathrm{~ms}$, aquisição de 125 pontos por varredura, e com uma resolução de $6,20 \mathrm{~nm} .{ }^{130}$

As amostras foram analisadas a partir do acessório de transmitância (Figura 2) e foi utilizada uma cubeta de quartzo de $2 \mathrm{~mL}$ com um caminho óptico de $1 \mathrm{~cm}$. A leitura do espectro de referência é feita com a cubeta vazia.

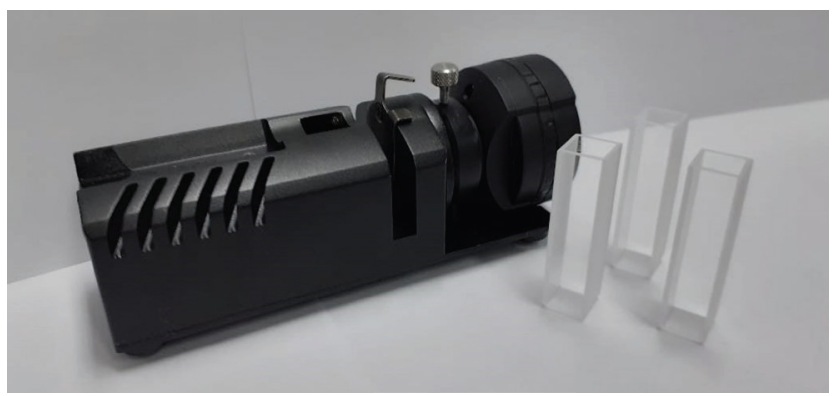

Figura 2. Acessório para análise de líquidos em cubeta acoplado ao MicroNIR

\section{Análise por Componentes Principais (PCA)}

Ao todo, 92 amostras foram analisadas em triplicata e para a construção dos modelos utilizou-se o espectro médio. Como os espectros NIR normalmente apresentam desvios na linha de base e efeitos de espalhamento de luz, ${ }^{133}$ os espectros foram tratados com o algoritmo de Savitzky-Golay com primeira derivada, janela de sete pontos e polinômio de ajuste de segunda ordem. ${ }^{134} \mathrm{Na}$ construção dos modelos PCA e PLS os espectros ainda foram centrados na média. O conjunto de 92 amostras correspondem a 12 amostras de AOEV, 6 de óleos compostos, 1 de óleo de soja e 19 das misturas preparadas de AOEV e óleo vegetal. As outras 60 amostras correspondem às amostras apreendidas pela PC-ES.
Na PCA, os dados são projetados em menores dimensões, calculadas por combinações lineares (componentes principais, PC), em particular, a partir dos valores de absorbância para cada comprimento de onda. As duas primeiras PC's foram responsáveis pela maior variabilidade total dos dados originais e foram avaliados em função do teor de adulteração (óleo vegetal adicionado ao AOEV).

\section{PLS}

Modelos PLS foram construídos para a curva da mistura AOEV/ óleo de soja no modo transmitância. O PLS foi usado para determinar o teor de óleo de soja na mistura com AOEV, sendo utilizado o procedimento de repeated double cross validation (RDCV). ${ }^{135} \mathrm{O}$ número correto de variáveis latentes foi determinado pelo procedimento k-fold, utilizando k igual a 5. Durante o procedimento RDCV, aproximadamente $25 \%$ do total de amostras foram separadas em cada interação. Em cada interação foi tomado o cuidado para que todas as réplicas de uma amostra ficassem no mesmo conjunto, seja calibração ou validação. O erro quadrado médio de predição (RMSEP) foi determinado conforme Equação 4.

$$
R M S E P=\sqrt{\frac{1}{\text { npred }} \sum(y i-\hat{y} i)^{2}}
$$

Em que npred é o número de amostras utilizadas e ŷi é o valor previsto pelo modelos PLS (teor de óleo vegetal em AOEV) do conjunto de dados de 19 concentrações da mistura AOEV/óleo vegetal. Os valores de viés, $\mathrm{R}^{2}$, LD e LQ foram calculados utilizando as Equações 5 a $8 .{ }^{136,137}$

$$
\begin{gathered}
\text { viés }=\sum_{i=1}^{n} \frac{y_{i}-\hat{y}_{i}}{N} \\
R^{2}=1-\frac{\sum\left(y_{i}-\hat{y}_{i}\right)^{2}}{\sum\left(y_{i}-\bar{y}_{i}\right)^{2}} \\
L D=3,3 \delta_{x}\left\|b_{k}\right\| \\
L Q=10 \delta_{x}\left\|b_{k}\right\|
\end{gathered}
$$

em que $b_{k}$ é o coeficiente de regressão do modelo PLS para k-variável com base no modelo de regressão e $\delta_{x}$ corresponde a estimativa do ruído instrumental, determinado como a variância média da matriz de resíduos instrumental. ${ }^{134}$

\section{RESULTADOS E DISCUSSÃO}

\section{Análises físico-químicas}

De uma maneira geral, os resultados das análises físico-químicas das amostras comerciais evidenciaram que para os testes de acidez e índice de peróxido todas as amostras se apresentaram dentro da conformidade. No entanto, algumas amostras demonstraram não conformidades mediante o teste de extinção específica, que analisa comprimentos de onda no UV-VIS.

O resultado para o teste de acidez está apresentado na Figura 3(a). Embora todas as amostras apresentassem conformidade no que diz respeito ao limite estabelecido para o índice de acidez ( $0,8 \%$ ácido oleico), as quais apresentaram valores de 0,13 - 0,36 wt \% ácido oleico, algumas amostras das marcas C (C1, C3 e C5) e E (E2, E6, E7, E9, E11 e E12) encontraram-se acima do valor de acidez, 0,2 wt\% ácido oleico, descrito em seus rótulos. 
No que tange a análise do índice de peróxido, os valores variaram de 1,44 - 13,33 meq/kg. A legislação estabelece um limite de $20 \mathrm{meq} / \mathrm{kg}$ para AOEV. Como apresentado na Figura 3(b), os valores encontram-se dentro do estabelecido. Assim, a presença de peróxidos e outros compostos com semelhanças químicas, resultantes da oxidação dos ácidos graxos insaturados são observadas em meq $\mathrm{O}_{2} / \mathrm{kg}$ (miliequivalente de oxigênio ativo por quilograma).

Em relação à análise da absorbância no ultravioleta, os valores, como apresentado na Figura 4, variaram de 0,396 - 2,649 para $\mathrm{K}_{270}$, $1,470-2,820$ para $K_{232}$ e $-1,894-0,498$ para $\Delta K$. Todas as amostras se encontram fora dos padrões estabelecidos, tendo em vista, que segundo a legislação referente ao AOEV, os limites para os valores $\mathrm{K}_{270}, \mathrm{~K}_{232}$ e $\Delta \mathrm{K}$ são de $0,22,2,5$ e 0,01 , respectivamente.

A análise de absorção no ultravioleta indica a presença de trienos conjugados e produtos de oxidação secundária $(270 \mathrm{~nm})$ assim como a presença de compostos carboxílicos, dienos conjugados e hidroperóxidos de ácido linoleico (232 nm). Um alto valor de absorção nesses comprimentos de onda evidencia a presença de óleos refinados, tendo em vista que o processo do refino é responsável por formar, a partir de ácidos graxos insaturados, compostos dienos e trienos conjugados. ${ }^{138}$

Portanto, tanto para o teste de extinção específica como para os outros parâmetros, o azeite só pode ser classificado como extra virgem quando este atender a todos os limites de tolerância de qualidade estabelecidos. Caso contrário, o azeite é classificado como fora de tipo e não pode ser comercializado como sendo extra virgem. ${ }^{8}$

Os valores detalhados dos resultados obtidos para as análises de acidez, peróxido e absorção no ultravioleta, também se encontram nas Tabelas 1S-3S, disponíveis no material suplementar.

\section{Espectroscopia NIR portátil}

A Figura 5 traz os espectros NIR brutos (Figura 5a) e após a aplicação da primeira derivada (Figura 5b) para as amostras de azeite apreendidas, AOEV comerciais, óleos compostos e óleo de soja. Note que, em geral, os espectros são bastante semelhantes, não sendo possível uma diferenciação visual do adulterante. Por isso, aplicam-se modelos quimiométricos, para a maximização das diferenças que possibilitem a identificação de padrões na identidade química das diferentes matrizes.

No perfil espectroscópico, observa-se as principais bandas de absorção localizadas em duas regiões: de 1100 a $1300 \mathrm{~nm}$ e 1350 a $1550 \mathrm{~nm}$. A região de 1100 - $1300 \mathrm{~nm}$ é característica do segundo sobretom de vibrações das ligações $\mathrm{C}-\mathrm{H}$, referentes aos grupos funcionais $-\mathrm{CH}_{2},-\mathrm{CH}_{3} \mathrm{e}-\mathrm{CH}=\mathrm{CH}-{ }^{139,140}$ Essas bandas são coerentes com a composição química formada por triacilgliceróis, ácidos graxos, entre outros. Em um estudo feito por Hourant et al. em óleos e gordura, percebeu-se que os óleos ricos em ácidos graxos insaturados apresentaram uma absorção mais evidente próximo a 1164 nm. Também foi relatado que a absorção na região de 1180 está relacionada com o grupo C-H de ácidos graxos puros contendo ligações duplas cis. ${ }^{141} \mathrm{~A}$ região de 1390 - 1490 nm está relacionada à combinação de vibração de estiramento C-H. ${ }^{142}$

Para identificar padrões nas amostras, o modelo PCA foi construído, a partir dos espectros NIR das amostras apreendidas juntamente com amostras de outros AOEV comerciais, óleos compostos e uma amostra de óleo de soja (Figura 6a). Podemos observar que as amostras de azeite apreendidas se agrupam na região de $\mathrm{PC} 1>0$, distantes das demais marcas comerciais que são classificadas como AOEV. Destaca-se também que a amostra de óleo de soja está na mesma região da PC1>0. Isso indica semelhança química entre as amostras, onde o gráfico de loadings (Figura 6b) evidencia a região de 1100 a 1150 nm e 1200 a 1300 responsável pela variância dessas amostras (regiões que caracterizadas pelo segundo sobretom de vibrações das ligações $\mathrm{C}-\mathrm{H}$ ).

Após a observação de padrões no perfil químico dos loadings da PCA das amostras apreendidas, o modelo de regressão PLS foi
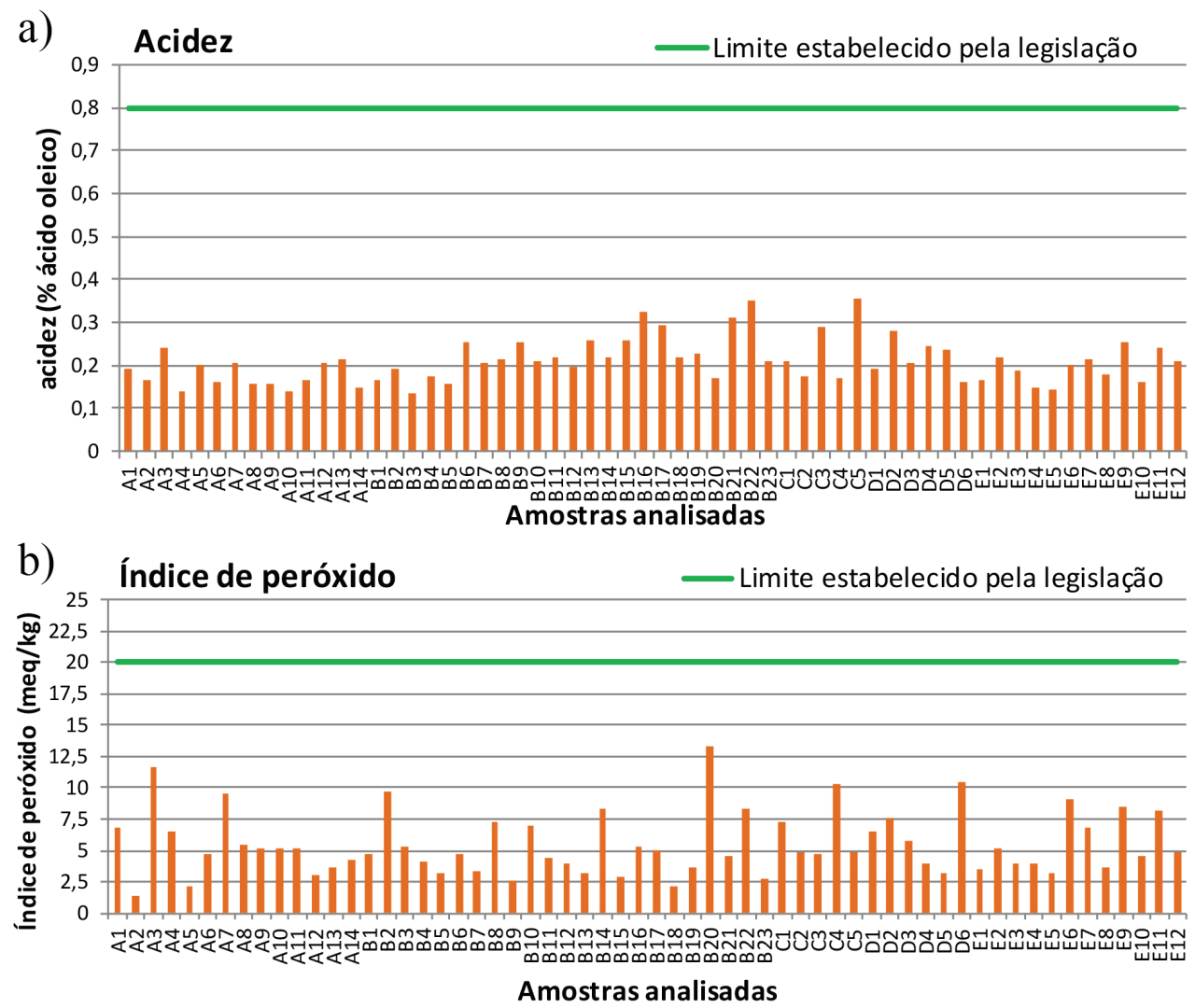

Figura 3. Valores experimentais para as análises de (a) acidez e (b) peróxido realizadas nas amostras apreendidas 

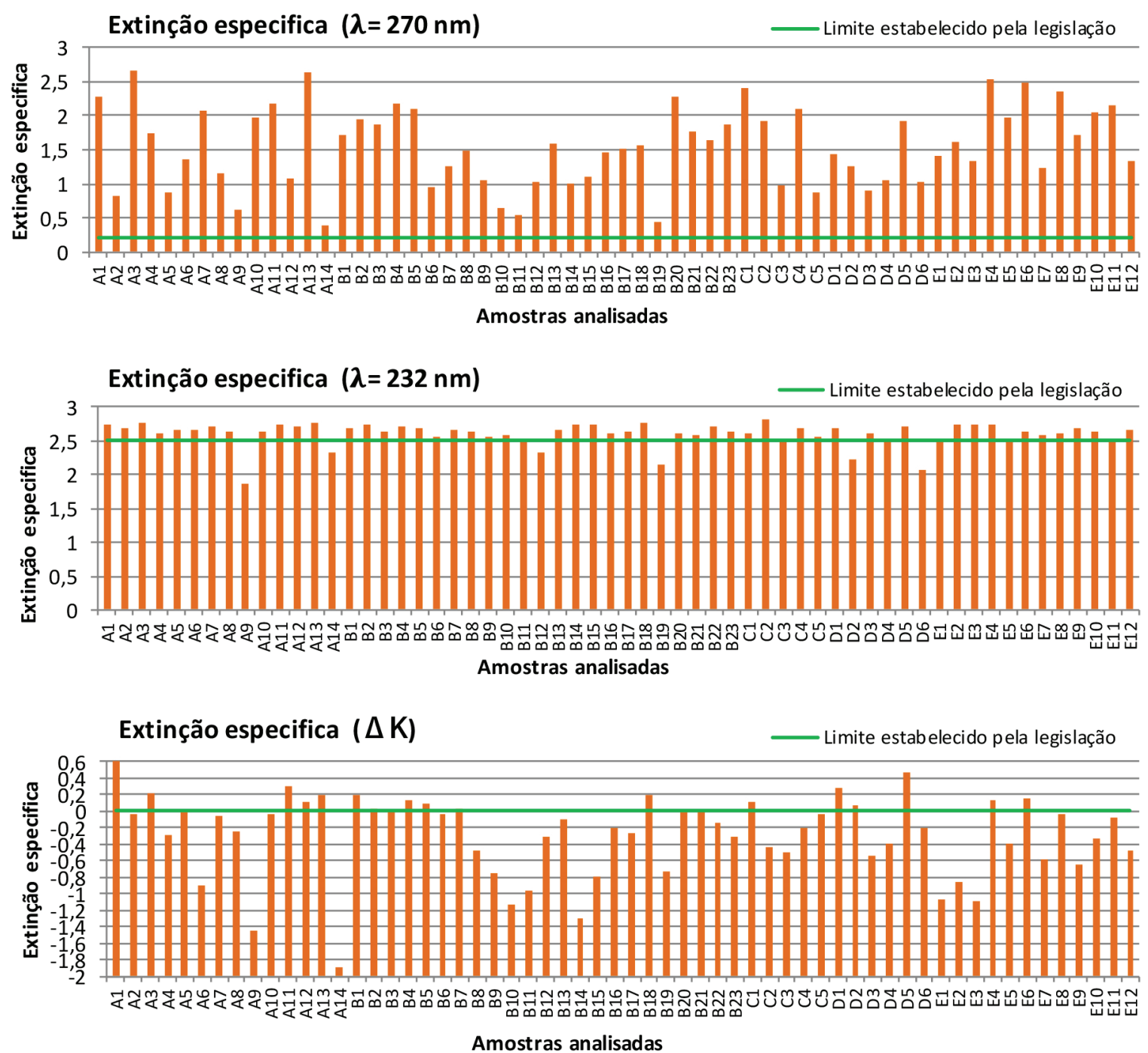

Figura 4. Resultado das análises da absorbância no ultravioleta, com os valores de extinção específica nos comprimentos de onda de 270 e 232 nm assim como $\triangle$ K calculado
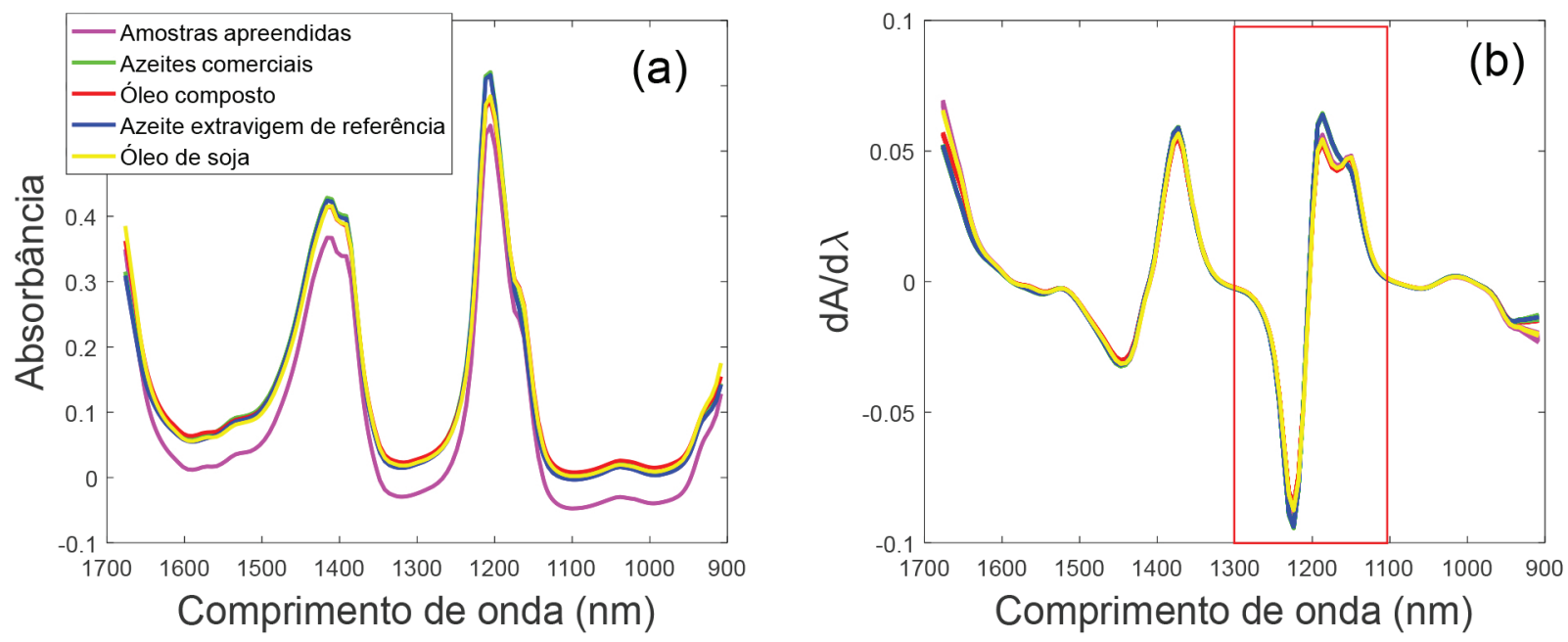

Figura 5. Espectros de infravermelho na região do proximo para as amostras de azeites apreendidas (a) brutos, e (b) tratados com a primeira derivada

construído para estimar o teor de óleo de soja nas amostras apreendidas. Para isso, foram utilizadas 19 blendas de óleo de soja em AOEV (com valores de 0 a 100 wt\%), o modelo PLS construído com duas variáveis latentes (Figura 7) apresentou um erro quadrático médio de previsão (RMSEP) de 4,8 wt\% e um coeficiente de determinação $\left(\mathrm{R}^{2}\right)$ de 0.975 , viés do modelo de 0,08 wt\% e valores de LD e LQ de $1,0 \mathrm{wt} \%$ e $3,4 \mathrm{wt} \%$ respectivamente (Figura 7a). Os valores de intervalo com $95 \%$ de confiança (IC95) das estimativas variam de 

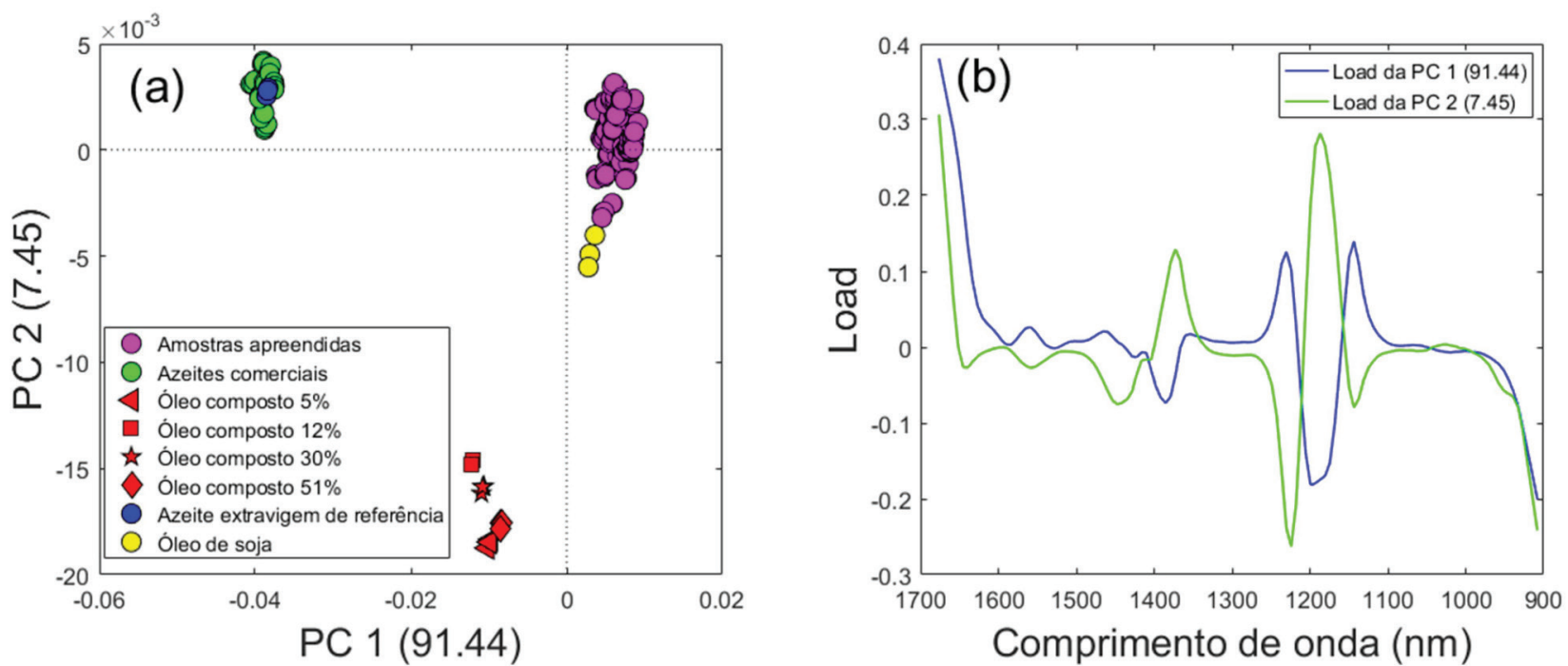

Figura 6. Gráficos de Scores para amostras de azeite apreendidas juntamente com amostras de óleo composto, AOEV comerciais e óleo de soja, e os (b) loadings referentes as $P C$ 's 1 e 2

6,9 a 9,8 wt\%, valores acima do LQ. Devido ao IC95 ser 6,9 wt\% para o ponto inicial da curva, este modelo não se aplica para valores de adulteração abaixo de 7 wt \% . O valor de viés apresentou ser não significativo no teste-t ao nível de significância de 5\%. O valor de $\mathrm{R}^{2}$ mostra uma boa relação dos valores estimados pelo PLS com os de referência. A elipse de confiança contém o ponto ideal $(1,0)$ confirmando a validade modelo é válido (Figura 7b).

As amostras de azeite apreendidas foram aplicadas ao modelo de quantificação construído, sendo que, a partir dos valores preditos (Tabela 3), observa-se que todas as amostras apresentaram alto teor de óleo de soja presente. Entre as amostras, apenas quatro (B1, B9, B13 e B16) foram quantificadas com menos de $100 \mathrm{wt} \%$ de óleo de soja. O modelo PLS também foi aplicado para as amostras de AOEV (AZ) e óleo de soja (OS), e os valores encontrados foram $0,01 \%$ e $105,7 \mathrm{wt} \%$ de óleo de soja, respectivamente. Os valores estimados do teor de óleo de soja acima de 100 wt\% estão dentro do intervalo de $95 \%$ de confiança na estimativa $(9,8 \mathrm{wt} \%$ para $100 \%$ de óleo de soja).

Tanto as análises físico-químicas quanto as espectroscópicas apontaram adulteração nas amostras apreendidas. Apesar dos testes de acidez e índice de peróxido não estarem relacionados com

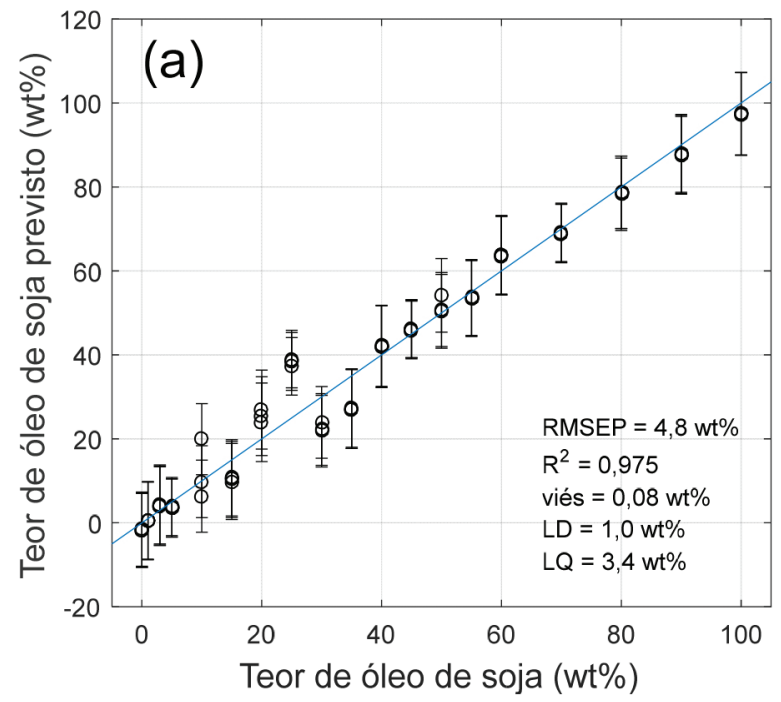

adulteração, estes evidenciaram que as amostras possuíam boa qualidade, resultado esperado devido às amostras estarem armazenadas há pouco tempo, não sofrendo alta exposição da luz, oxigênio ou umidade. Por outro lado, os resultados das análises de absorção no ultravioleta indicaram a presença de óleo refinado nas amostras analisadas, o que também é observado através dos resultados da PCA, em que as amostras apreendidas se agruparam com as amostras de óleo de soja (PC1>0) e da quantificação, na qual as amostras apresentaram alto teor desse adulterante.

\section{CONCLUSÃO}

Os resultados obtidos mostram que o MicroNIR, em conjunto com os modelos quimiométricos (PCA e PLS), mostrou-se uma ferramenta analítica eficaz na determinação da autenticidade das amostras de azeite apreendidas. Através do modelo PCA foi possível identificar as amostras como sendo AOEV autêntico; AOEV adulterado com $100 \%$ de óleo de soja (amostras apreendidas); e óleo composto (misturas de AOEV com óleo de soja). A partir do modelo PLS quantificou-se o teor de óleo de soja, encontrando valores de até $100 \mathrm{wt} \%$, na

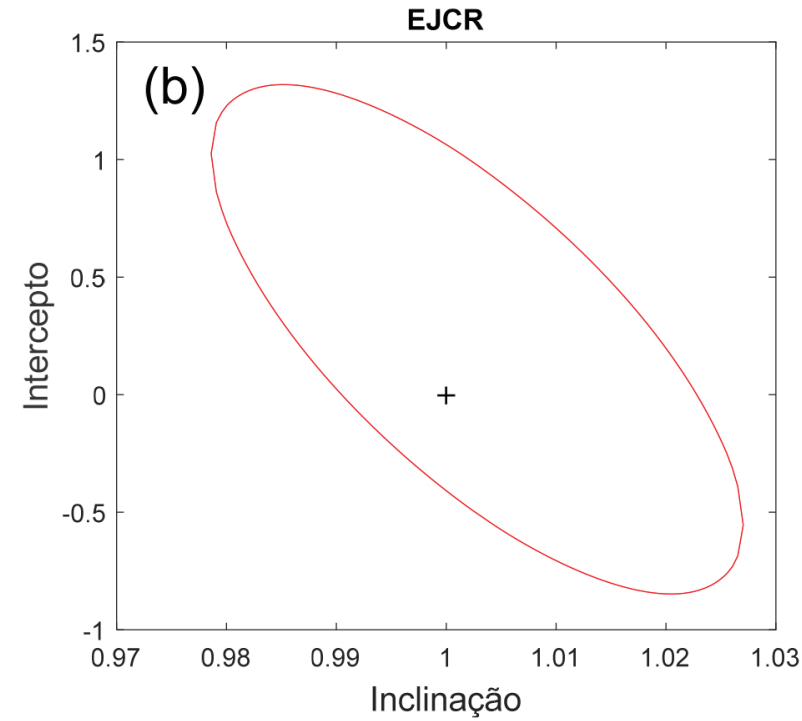

Figura 7. (a) Gráficos dos valores preditos e de referência de óleo de soja na curva de 40 a 100 wt\% em AOEV/óleo de soja com $95 \%$ de confiança. (b) elipse de confiança EJCR 
Tabela 3. Valores de previsão de óleo de soja presente nas amostras de azeites aprendidas pela PC-ES aplicado ao modelo PLS

\begin{tabular}{cccccc}
\hline COD & $\begin{array}{c}\text { \% de óleo } \\
\text { prevista }\end{array}$ & COD & $\begin{array}{c}\text { \% de óleo } \\
\text { prevista }\end{array}$ & COD & $\begin{array}{c}\text { \% de óleo } \\
\text { prevista }\end{array}$ \\
\hline A1 & 104,2 & B8 & 102 & D1 & 102,1 \\
A2 & 105,8 & B9 & 97,9 & D2 & 101,7 \\
A3 & 101,5 & B10 & 100,5 & D3 & 107 \\
A4 & 104,8 & B11 & 103,6 & D4 & 107,6 \\
A5 & 106,5 & B12 & 103,5 & D5 & 107,6 \\
A6 & 103,7 & B13 & 97,6 & D6 & 101,5 \\
A7 & 104,2 & B14 & 101,6 & E1 & 109,1 \\
A8 & 100,9 & B15 & 100,8 & E2 & 102,5 \\
A9 & 105,3 & B16 & 98,5 & E3 & 108,9 \\
A10 & 104,1 & B17 & 104,9 & E4 & 109,2 \\
A11 & 104,3 & B18 & 103,5 & E5 & 109,1 \\
A12 & 106,6 & B19 & 101,1 & E6 & 107,6 \\
A13 & 104,5 & B20 & 108,5 & E7 & 102,9 \\
A14 & 104,1 & B21 & 104,4 & E8 & 109,1 \\
B1 & 97,3 & B22 & 104,5 & E9 & 106,8 \\
B2 & 101,9 & B23 & 101,5 & E10 & 109,1 \\
B3 & 105,3 & C1 & 109,4 & E11 & 107,6 \\
B4 & 101,8 & C2 & 109,2 & E12 & 102,3 \\
B5 & 101,6 & C3 & 104,6 & AZ & 0,01 \\
B6 & 100,9 & C4 & 108,9 & OS & 105,7 \\
B7 & 100,2 & C5 & 104,4 & & \\
\hline & & & & & \\
\hline
\end{tabular}

maior parte dos casos. Comparando esses dados aos resultados das análises físico-químicas, todas as amostras foram classificadas como não sendo de óleo extra virgem, apresentando não conformidade para a propriedade de extinção específica.

\section{AGRADECIMENTOS}

Os autores agradecem à CAPES (23038.007083/2014-40), FAPES (EDITAL CNPq/FAPES No 23/2018 - PRONEM (596/ 2018) e edital EDITAL FAPES No 11/2018 - - PIBICES 2018), e ao CNPq (422555/2018-5, e 305359/2017-7) pelo suporte financeiro. Os autores também gostariam de agradecer ao Núcleo de Competências em Química do Petróleo e ao LabPetro pelo uso de suas instalações e a Polícia Civil do Espírito Santo pelo fornecimento das amostras.

\section{MATERIAL SUPLEMENTAR}

Algumas imagens dos sistemas utilizados neste trabalho estão disponíveis, para melhor compreensão, em http://quimicanova.sbq. org.br, na forma de arquivo PDF, com acesso livre.

\section{REFERÊNCIAS}

1. Peng, G.; Chang, M.; Fang, M.; Liao, C.; Tsai, C.; Tseng, S.; Kao, Y.; Chou, H.; Cheng, H.; Food Control 2017, 72, 145.

2. Kowalska, A.; Acta Univ. Agric. Silvic. Mendelianae Brun. 2018, 66, 1275 .

3. Manning, L.; Curr. Opin. Food Sci. 2016, 10, 16.

4. Kalivas, J. H.; Georgiou, C. A.; Moira, M.; Tsafaras, I.; Eleftherios A. P.; Mousdis, G. A.; Food Chem. 2014, 148, 289.
5. Danezis, G. P.; Tsagkaris, A. S.; Brusic, V.; Georgiou, C. A.; Curr. Opin. Food Sci. 2016, 10, 22.

6. Van Durme, J.; Vandamme, J.; Food Chem 2016, 208, 185.

7. http://bvsms.saude.gov.br/bvs/saudelegis/anvisa/2005/ rdc0270_22_09_2005.html, acessada em Maio 2020.

8. https://azeiteonline.com.br/2012/02/02/publicado-o-regulamentotecnico-do-azeite-do-mapa/, acessada em Maio 2020.

9. https://tribunadejundiai.com.br/gastronomia/governo-proibe-venda-de32-marcas-de-azeites-fraudados/, acessada em Maio de 2020

10. Aued-Pimentel, S.; Separovic L.; Silvestre, L. G. G. R.; Kus-Yamashita, M. M. M.; Takemoto, E.; Vigilância Sanitária em Debate: Sociedade, Ciência \& Tecnologia 2017, 5, 84.

11. Stefano, V. di; Avellone, G.; Bongiorno, D.; Cunsolo, V.; Muccilli, V.; Sforza, S.; Dossena, A.; Drahos, L.; Vékey, K.; J. Chromatogr. A 2012, $1259,74$.

12. Guo, M.; He, M.; Zhong, J.; He, Q.; Ismail, B. B.; Chen, G.; Liu, D.; Sci. Total Environ. 2020, 710, 134975.

13. Shishov, A.; Gagarionova, S.; Bulatov, A.; Food Chem. 2020, 314, 126097.

14. Saridal, K.; Ulusoy, H. I.; Microchem. J. 2019, 150, 104170.

15. Thalhamer, B.; Buchberger, W.; Food Control 2019, 105, 58.

16. Chajduk, E.; Polkowska-Motrenko, H.; Food Chem. 2019, 292, 129.

17. Xie, X.; Zhang, Y.; Yue, Z.; Wang, K.; Mai, X.; Liu, Y.; Zhu, M.; Fan, H.; Zhang, W.; Microchem. J. 2019, 149, 103995.

18. Lim, H. S.; Hwang, J. Y.; C, E.; Lee, G.; Yoon, S. S.; Kim, M.; Food Chem. 2018, 239, 1167.

19. Michalak, J.; Gujska, E.; Kuncewicz, A.; J. Food Compos. Anal. 2013, 32,68 .

20. Luterotti, S.; Marković, K.; Franko, M.; Bicanic, D.; Madžgalj, A.; Kljak, K.; Food Chem. 2013, 140, 390.

21. Brera, C.; Debegnach, F.; De Santis, B.; Pannunzi, E.; Berdini, C.; Prantera, E.; Gregori, E.; Miraglia, M.; Talanta 2011, 83, 1442.

22. Nie, Q.; Nie, S. In Evaluation Technologies for Food Quality; Zhong, J., Wang, X., eds.; Woodhead Publishing: Cambridge, 2019, cap. 13.

23. Santos, I. C.; Smuts, J.; Schug, K. A.; Food Anal. Methods 2017, 10, 4068.

24. Ŝnidertič, L.; Mlakar, A.; Prosen, H.; Food Chem. Toxicol. 2019, 134, 110829.

25. Bueno, M.; Resconi, V. C.; Campo, M. M.; Ferreira, V.; Escudero, A.; Food Chem. 2019, 281, 49.

26. Rocchi, R.; Mascini, M.; Faberi, A.; Sergi, M.; Compagnone, D.; Di Martino, V.; Carradori, S.; Pittia, P.; Food Control 2019, 106, 106736.

27. Svečnjak, L.; Jović, O.; Prđun, S.; Rogina, J.; Marijanović, Z.; Car, J.; Matošević, M.; Jerković, I.; Food Chem. 2019, 291, 187

28. Feng, T.; Sun, M.; Song, S.; Zhuang, H.; Yao, L. In Evaluation Technologies for Food Quality 2019, Woodhead Publishing Series in Food Science, Technology and Nutrition: Cambdridge, pp. 219-265.

29. Torrecilla, J. S.; Rojo, E.; Dominguez, J. C.; Rodriguez, F.; J. Agric. Food Chem. 2010, 58, 1679.

30. Anibal, C. V. di; Ruisánchez, I.; Fernández, M.; Forteza, R.; Cerdà, V.; Callao, M. P.; Food Chem. 2012, 134, 2326.

31. Vera, D. N.; Ruisánchez, I.; Callao, M. P.; Food Control 2018, 92, 341.

32. Márquez, C.; Ruisánchez, I.; Callao, M. P.; Microchem. J. 2019, 145, 686.

33. Anibal, C. V. di; Callao, M. P.; Ruisánchez, I.; Talanta 2011, 84, 829.

34. Anibal, C. di; Rodríguez, S.; Albertengo, L.; Rodríguez, M.; Food Anal. Methods 2016, 9, 3117

35. Anibal, C. di; Rodriguez, M. S.; Albertengo, L.; Food Anal. Methods 2013, 7, 1090.

36. Riovanto, R.; Marchi, M. de; Cassandro, M.; Penasa, M.; Food Chem. 2012, 134, 2459

37. Barbin, D. F.; Felicio, A. L. de S. M.; Sun, D.; Nixdorf, S. L.; Hirooka, E. Y.; Food Res. Int. 2014, 61, 23. 
38. Sørensen, K. M.; Khakimov, B.; Engelsen, S. B.; Curr. Opin. Food Sci. 2016, $10,45$.

39. Rungpichayapichet, P.; Mahayothee, B.; Nagle, M.; Khuwijitjaru, P.; Müller, J.; Postharvest Biol. Technol. 2016, 111, 31.

40. Xu, L.; Shi, W.; Cai, C.; Zhong, W.; Tu, K.; LWT--Food Sci. Technol. 2015, 61, 590 .

41. Márquez, C.; López, M. I.; Ruisánchez, I.; Callao, M. P.; Talanta 2016, 161,80

42. Pullanagari, R. R.; Yule, I. J.; Agnew, M.; Meat Sci. 2015, 100, 156.

43. Pedreschi, F.; Segtnan, V. H.; Knutsen, S. H.; Food Chem. 2010, 121, 616.

44. Sánchez, M.; Haba, M. J. de la ; Benítez-López, M.; Fernández-Novales, J.; Garrido-Varo, A.; Pérez-Marín, D.; J. Food Eng. 2012, 110, 102.

45. López, M. I.; Trullols, E.; Callao, M. P.; Ruisánchez, I.; Food Chem. 2014, 147, 177.

46. Kamruzzaman, M.; Sun, D.; Elmasry, G.; Allen, P.; Talanta 2013, 103, 130.

47. Núñez-Sánchez, N.; Martínez-Marín, A. L.; Polvillo, O.; FernándezCabanás, V. M.; Carrizosa, J.; Urrutia, B.; Serradilla, J. M.; Food Chem. 2016, 190, 244

48. Pan, L.; Zhu, Q.; Lu, R.; Mcgrath, J. M.; Food Chem. 2015, 167, 264.

49. Chen, L.; Wang, J.; Ye, Z.; Zhao, J.; Xue, X.; Heyden, Y. V.; Sun, Q.; Food Chem. 2012, 135, 338.

50. Mishra, P.; Cordella, C. B.Y.; Rutledge, D. N.; Barreiro, P.; Roger, J. M.; Diezma, B.; J. Food Eng. 2016, 168, 7.

51. Huang, L.; Zhao, J.; Chen, Q.; Zhang, Y.; Food Chem. 2014, 145, 228.

52. Teye, E.; Anyidoho, E.; Agbemafle, R.; Sam-Amoah, L. K.; Elliott, C.; Infrared Phys. Technol. 2020, 104, 103.

53. Bosco, G. L.; James, L.; TrAC, Trends Anal. Chem. 2010, 29, 197.

54. Santos, C. A. T. dos; Páscoa, R. N. M. J.; Lopes, J. A.; TrAC, Trends Anal. Chem. 2017, 88, 100.

55. Rohman, A.; Man, Y. B. C.; Food Chem. 2011, 129, 583.

56. Georgouli, K.; Rincon, J. M. del; Koidis, A.; Food Chem. 2017, 217, 735.

57. Horn, B.; Esslinger, S.; Pfister, M.; Fauhl-Hassek, C.; Riedl, J., Food Chem. 2018, 257, 112

58. Botelho, B. G.; Reis, N.; Oliveira, L. S.; Sena, M. M.; Food Chem. 2015 $181,31$.

59. Koca, N.; Kocaoglu-Vurma, N. A.; Harper, W. J.; Rodriguez-Saona, L. E.; Food Chem. 2010, 121, 778.

60. Liu, J.; Wen, Y.; Dong, N.; Lai, C.; Zhao, G.; Food Chem. 2013, 141, 3103.

61. Miaw, C. S. W.; Sena, M. M.; Souza, S. V. C. de; Callao, M. P.; Ruisanchez, I.; Food Chem. 2018, 266, 254

62. Wu, Z.; Xu, E.; Long, J.; Wang, F.; Xu, X.; Jin, Z.; Jiao, A.; J. Food Sci. 2015, 80, 1670.

63. Xu, M.; Gao, Y.; Han, X. X.; Zhao, B.; J. Agric. Food Chem. 2017, 65 6719.

64. Mateescu, C.; Popescu, A. M.; Radu, G. L.; Onisei, T.; Raducanu, A. E.; Adv. Pharm. Bull. 2017, 7, 251.

65. Lee, J.; Park, J.; Mun, H.; Shim, W.; Lim, S.; Kim, M.; Food Chem. 2018, 254, 109.

66. Gukowsky, J. C.; Xie, T.; Gao, S.; Qu, Y.; He, L.; Food Control 2018 92, 267.

67. Nedeljkivić, A.; Rösch, P.; Popp, J.; Miočinović, J.; Radovanović, M.; Pudja, P.; Food Anal. Methods 2015, 9, 1315.

68. Khalil, I.; Yehye, W. A.; Julkapli, N. M.; Sina, A. A. I.; Rahmati, S.; Basirun, W. J.; Seyfoddin, A.; The Analyst 2020, 145, 1414

69. Craig, A.; Franca, A.; Irudayaraj, J.; Annu. Rev. Food Sci. Technol 2013, 4,369 .

70. Lohumi S.; Lee H.; Kim M. S.; Qin J.; Kandpal L. M.; Bae H.; Rahman, A.; Cho, B.; PloS One 2018, 13, 1.

71. Tintchev, F.; Wackerbarth, H.; Kuhlmann, U.; Toepfl, S.; Knorr, D.;
Hildebrandt, P.; Heinz, V.; Annu. N. Y. Acad. Sci. 2010, 1189, 34.

72. Wang, H.; Guo, X.; Fu, S.; Yang, T.; Wen, Y.; Yang, H.; Food Chem. 2015, 188, 137

73. Fan, C.; Hu, Z.; Riley, L. K.; Purdy, G. A.; M, A.; Lin, M.; J. Food Sci. 2010, 302

74. Mangolim, C. S.; Moriwaki, C.; Nogueira, A. C.; Sato, F.; Baesso, M. L.; Neto, A. M.; Matioli, G.; Food Chem. 2014, 153, 361.

75. Guyader, S.; Thomas, F.; Portaluri, V.; Jamin, E.; Akoka, S.; Silvestre, V.; Remaud, G.; Food Control 2018, 91, 216.

76. Tomassini, A.; Capuani, G.; Delfini, M.; Miccheli, A.; Data Handl. Sci. Technol. 2013, 28, 441

77. Bo, Y.; Feng, J.; Xu, J.; Huang, Y.; Cai, H.; Cui, X.; Dong, J.; Ding, S.; Chen, Z.; Food Res. Int. 2019, 125, 108574

78. Trimigno, A.; Marincola, F. C.; Dellarosa, N.; Picone, G.; Laghi, L.; Curr. Opin. Food Sci. 2015, 4, 99.

79. Palaric, C.; Pilard, S.; Fontaine, J.; Cailleu, D.; Mesnard, F.; Renaud, T.; Petit, A.; Molinié, R.; Food Control 2018, 88, 217.

80. Sobolev, A. P.; Thomas, F.; Donarski, J.; Ingallina, C.; Circi, S.; Marincola, F. C.; Capitani, D.; Mannina, L.; Trends Food Sci. Technol. 2019, 91, 347.

81. Minoja, A. P.; Napoli, C.; Food Res. Int. 2014, 63, 126.

82. Marcone, M. F.; Wang, S.; Albabish, W.; Nie, S.; Somnarain, D.; Hill, A.; Food Res. Int. 2013, 51, 729.

83. Siudem, P.; Wawer, I.; Paradowska, K.; J. Mol. Struct. 2019, 1177, 204

84. Cai, S.; Zang, Y.; Xia, F.; Shen, G.; Feng, J.; J. Food Compos. Anal. 2019, 84, 103316

85. Consonni, R.; Cagliani, L. R.; Adv. Food Nutr. Res. 2010, 59, 87.

86. Duynhoven, J.; Voda, M.; Witek, M.; As, H.; Annu. Rep. NMR Spectrosc. 2010, 69, 145

87. Ghasemi-Varnamkhasti, M.; Mohtasebi, S. S.; Rodriguez-Mendez, M. L.; Lozano, J.; Razavi, S. H.; Ahmadi, H.; Trends Food Sci. Technol. 2011, 22, 165

88. Chen, Q.; Zhao, J.; Chen, Z.; Lin, H.; Zhao, D.; Sens. Actuators, B 2011 159, 294.

89. Hong, X.; Wang, J.; Hai, Z.; Sens. Actuators, B 2012, 161, 381.

90. Qiu, S.; Gao, L.; Wang, J.; J. Food Eng. 2015, 144, 77.

91. Sanaeifar, A.; Mohtasebi, S. S.; Ghasemi-Varnamkhasti, M.; Ahmadi, H.; Measurement 2016, 82, 105.

92. Lohumi, S.; Lee, S.; Lee, H.; Cho, B.; Trends Food Sci. Technol. 2015, 46,85 .

93. Sato, T.; J. Am. Oil. Chem. Soc. 1994, 71, 293.

94. Wesley, I. J.; Pacheco, F.; McGill, A. E. J; J. Am. Oil. Chem. Soc. 1996, 73,515 .

95. Hein, M.; Henning,H.; Isengard, H.; Talanta 1998, 47, 447.

96. Downey, G.; Mcintyre, P.; Davies, A. N.; J. Agric. Food Chem. 2002, 50, 5520.

97. Cozzolino, D.; Murray, I.; Chree, A.; Scaife, J. R.; Food Sci. Technol. 2005, 38, 821 .

98. Yang, H.; Irudayaraj, J.; Paradkar, M.; Food Chem. 2005, 93, 25

99. Pereira, A. F. C.; Pontes, M. J. C.; Gambarra Neto, F. F.; Santos, S. R. B.; Galvão, R. K, H.; Araðjo, M. C. U.; Food. Res. Int. 2008, 41, 341.

100. Cayuela, J.; García, J. F.; J. Food Eng. 2017, 202, 79.

101. Chen, H.; Lin, Z.; Tan, C.; Vib. Spectrosc. 2018, 99, 178

102. Picouet, P. A.; Gou, P.; Hyypiö, R.; Castellari, M.; J. Food Eng. 2018 , $230,18$.

103. Farres, S.; Srata, L.; Fethi, F.; Kadaoui, A.; Vib. Spectrosc. 2019, 102, 79.

104. Mustorgi, E.; Malegori, C.; Oliveri, P.; Hooshyary, M.; Bounnece, H.; Mondello, L.; Oteri, M.; Casale, M.; Chemometr. Intell. Lab. 2020, 199, 103974

105. Rodriguez-Saona, L. E.; Giusti, M. M.; Shotts, M. In Advances in Food Authenticity Testing; Downey, G., ed.; Woodhead Publishing: Cambridge, 2016, cap. 4. 
106. Ozaki, Y.; Genkawa, T.; Futami, Y.; In Encyclopedia of Spectroscopy and Spectrometry; Lindon, J. C.; Tranter, G. E.; Koppenaal, D. W. eds.; Academic Press: Cambridge, 2017.

107. Manley, M.; Baeten, V. In Modern Techniques for Food Authentication; Sun, D. ed.; Academic Press: Cambridge, 2018, cap. 3.

108. Qu, J.; Liu, D.; Cheng, J.; Sun, D.; Ma, J.; Pu, H.; Zeng, X.; Crit. Rev. Food Sci. Nutr. 2015, 55, 1939.

109. Inarejos-García, A. M.; Gómez-Alonso, S.; Fregapane, G.; Salvador, M. D.; Food Res. Int. 2013, 50, 250.

110. Pereira, C. G.; Leite, A. I. N.; Andrade, J.; Bell, M. J. V.; Anjos, V.; Food Sci. Technol. 2019, 107, 1.

111. Özdemir, İ. S.; Dağ, Ç.; Özinanç, G.; Suçsoran, Ö.; Ertaş, E.; Bekiroğlu, S.; LWT--Food Sci. Technol. 2018, 91, 125.

112. Rambo, M. K. D.; Ferreira, Márcia M. C.; Rev. Virtual Quim. 2018, 10, 421.

113. Sinelli, N.; Casale, M.; Egidio, V. di; Oliveri, P.; Bassi, D.; Tura, D.; Casiraghi, E.; Food Res. Int. 2010, 43, 2126.

114. Karunathilaka, S. R.; Kia, A. F.; Srigley, C Chung, J. K.; Mossoba, M. M.; J. Food Sci. 2016, 81, 2390.

115. Vanstone, N.; Moore, A.; Martos, P.; Neethirajan, S.; Food Qual. Saf. 2018, 2, 189.

116. Willenberg, I.; MatthÄus, B.; Gertz, Christian.; Eur. J. Lipid. Sci. Tech. 2018, 121, 1800361.

117. Yan, J.; Stuijvenberg, L.; Ruth, S. M.; Eur. J. Lipid Sci. Technol. 2019, 121,1900031

118. Santos, C. A. T.; Lopo, M.; Páscoa, R. N. M. J.; Lopes, J. A.; Appl. Spectrosc. 2013, 67, 1215.

119. Malegori, C.; Marques, E. J. N.; De Freitas, S. T.; Pimentel, M. F.; Pasquini, C.; Casiraghi, E.; Talanta 2017, 165, 112.

120. Basri, K. N.; Hussain, M. N.; Bakar, J.; Sharif, Z.; Khir, M. F. A.; Zoolfakar, A. S.; Spectrochim. Acta A 2017, 173, 335.

121. Kaufmann, K. C.; Favero, F. F.; Vasconcelos, M. A. M.; Godoy, H. T.; Sampaio, K. A.; Barbin, D. F. J. Food Sci. 2019, 84, 406.

122. Oliveira, M. M.; Cruz-Tirado, J. P.; Roque, J. V.; Teófilo, R. F.; Barbin, D. F.; J. Food Compos. Anal. 2020, 87, 1.

123. Pan, L.; Lu, R.; Zhu, Q.; Mcgrath, J. M.; Tu, K.; Postharvest Biol. Technol. 2015, 102, 42 .

124. Correia, R. M.; Domingos, E.; Cáo, V. M.; Araújo, B. R. F.; Sena, S.; Pinheiro, L. U.; Fontes A. M.; Aquino, L. F. M.; Ferreira, E. C.; Filgueiras, P. R.; Romão, W.; Talanta 2018, 176, 26.
125. Liu, N.; Parra, H. A.; Pustjens, A.; Hettinga, K.; Mongondry, P.; van Ruth, S. M.; Talanta 2018, 184, 128.

126. Savoia, S.; Albera, A.; Brugiapaglia, A.; di Stasio, L.; Ferragina, A.; Cecchinato, A.; Bittante, G.; Meat Sci. 2020, 161, 108017.

127. Rios, H. C. S.; Pereira, I. R. O.; de Abreu, E. S.; Ciência \& Saúde 2013 , 6,118 .

128. Custódio, T. A. da S.; Dissertação de Mestrado, Faculdade de Farmácia da Universidade do Porto, Portugal, 2009.

129. Instituto Adolfo Lutz; Normas Analíticas do Instituto Adolfo Lutz: Métodos químicos e físicos para análise de alimentos, $3^{\text {th }}$ ed., IMESP: São Paulo, 1985.

130. https://eur-lex.europa.eu/legal-content/PT/TXT/?uri=CELEX\%3A0199 1R2568-20150101, acessada em Maio 2020

131. Cardoso, L. G. V.; Barcelos, M. de F. P.; Oliveira, A. F. de; Pereira, J. de A. R.; Abreu, W. C. de; Pimentel, F. A.; Cardoso, M. das G.; Pereira, M. C. de A.; Semina: Cienc. Agrar. 2010, 31, 127.

132. Peixoto, E. R. M.; Santana, D. M. N.; Abrantes, S.; Cienc. Tecnol. Aliment. 1998, 18, 444

133. Pasquini, C.; Anal. Chim. Acta 2018, 1026, 8.

134. Savitzky,A.; Golay, M. J. E.; Anal. Chem. 1964, 36, 1627

135. Filzmoser, P.; Liebmann, B.; Varmuza, K.; J. Chemometr. 2009, 23, 60.

136. Portela, N. A.; Oliveira, E. C. S.; Neto, A. C.; Rodrigues, R. R. T.; Silva, S. R. C.; Castro, E. V. R.; Filgueiras, P. R.; Fuel 2016, 166, 12.

137. Souza, A. M.; Filgueiras, P. R.; Coelho, M. R.; Fontana, A.; Winkler, T. C. B; Valderrama, P.; Poppi, R. J.; J. Near Infrared Spectrosc. 2016, 24, 293.

138. Aued-Pimentel, S.; Takemoto, E.; Minazzi-Rodrigues, R. S.; Badolato, E. S. G.; Rev. Inst. Adolfo Lutz, 2002, 61, 69.

139. Christy, A. A.; Kasemsumran, S.; Du, Y.; Ozaki,Y.; Anal. Sci. 2004, 20 , 935.

140. Sinelli, N.; Casale, M.; Egidio, V. di; Oliveri, P.; Bassi, D.; Tura, D.; Casiraghi, E.; Food Res. Int. 2010, 43, 2126.

141. Hourant, P.; Baeten, V.; Morales, M. T.; Meurens, M.; Aparicio, R.; Appl. Spectrosc. 2000, 54, 1168 .

142. Galtier, O.; Dupuy, N.; Le Dreau, Y. Ollivier, D.; Pinatel, C.; Kister, J.; Artaud, J.; Anal. Chim. Acta 2007, 595, 136 